Full length article

\title{
Early childhood adversity potentiates the adverse association between prenatal organophosphate pesticide exposure and child IQ: The CHAMACOS cohort
}

\author{
Lauren J. Stein, Robert B. Gunier, Kim Harley, Katherine Kogut, Asa Bradman, \\ Brenda Eskenazi, PhD* \\ Center for Environmental Research and Children's Health, School of Public Health, University of California, Berkeley, United States
}

\section{A R T I C L E I N F O}

\section{Article history:}

Received 22 March 2016

Received in revised form 20 July 2016

Accepted 21 July 2016

Available online 26 July 2016

\section{Keywords:}

Organophosphate pesticides

Pesticides

Early life adversity

In utero exposures

Child IQ

\begin{abstract}
A B S T R A C T
Background: Previous studies have observed an adverse association between prenatal exposure to organophosphate pesticide (OPs) and child cognition, but few studies consider the potential role of social stressors in modifying this relationship.

Objective: We seek to explore the potential role of early social adversities in modifying the relationship between OPs and child IQ in an agricultural Mexican American population.

Methods: Participants from the Center for the Health Assessment of Mothers and Children of Salinas (CHAMACOS) study, a prospective longitudinal pre-birth cohort study, include 329 singleton infants and their mothers followed from pregnancy through age 7. Dialkyl phosphate metabolite concentrations (DAPs), a biomarker of organophosphate pesticide exposure, were measured in maternal urine collected twice during pregnancy and averaged. Child cognitive ability was assessed at 7 years using the Wechsler Intelligence Scale for Children - Fourth Edition. Demographic characteristics and adversity information were collected during interviews and home visits at numerous time points from pregnancy until age 7 . Results: Among low-income Latina mothers and their children in the Salinas Valley, total adversity and specific domains of adversity including poor learning environment and adverse parent-child relationships were negatively associated with child cognition. Adverse associations between DAP concentrations and IQ were stronger in children experiencing greater adversity; these associations varied by child sex. For example, the association between prenatal OP exposure and Full-Scale IQ is potentiated among boys who experienced high adversity in the learning environment $(\beta=-13.3$; $p$-value $<0.01)$.

Conclusions: Greater total and domain-specific adversity modifies negative relationships between prenatal OP exposure and child IQ differently among male and female children. These findings emphasize the need to consider plausible interactive pathways between social adversities and environmental exposures.
\end{abstract}

(๖ 2016 Elsevier B.V. All rights reserved.

\section{Introduction}

Although most organophosphate (OP) pesticides are no longer allowed for residential use, they continue to be widely used in the United States for agricultural purposes. In 2012, more than 1.1 million kilograms of OP pesticides were used agriculturally in California alone (California Department of Pesticide Regulation, 2012). Use is steadily declining, but risks due to exposure for residents in agricultural communities remain relevant. OPs are

\footnotetext{
* Corresponding author at: School of Public Health, 1995 University Ave Suite 265, Berkeley, CA 94720, United States.

E-mail address: eskenazi@berkeley.edu (B. Eskenazi).
}

neurotoxicants, which at high doses in humans can cause coma and death, primarily by inhibition of the enzyme acetylcholinesterase (Sultatos, 1994). At lower doses, exposure to some OPs may affect second-messenger systems, growth factors, and the production, storage and receptors for neurotransmitters including serotonin, norepinephrine, acetylcholine, and dopamine (Slotkin and Seidler 2007; Verma et al., 2009). Several studies have suggested that low-dose OP exposure in utero is associated with poorer neurodevelopment in infants (Engel et al., 2007) and toddlers (Engel et al., 2007; Handal et al., 2008; Rauh et al., 2006). In the CHAMACOS cohort, we reported that prenatal maternal dialkyl phosphate (DAP) metabolites, a urinary biomarker of OP exposure, were significantly associated with children's mental development at 24 months as assessed by the Bayley Scales of 
Infant Development (Eskenazi et al., 2007) and with Full-Scale and subtest intelligence quotient (IQ) scores at 7 years, measured by Wechsler Intelligence Scale for Children (WISC-IV) (Bouchard et al., 2011).

There is growing evidence from animal studies suggesting that early life exposure to toxic stress may magnify the effects of environmental chemical exposure on neurodevelopment (Bellinger, 2008). For example, studies have shown that maternal stressors, such as restraint stress, synergistically interact with diesel exhaust (Bolton et al., 2013) and lead (Cory-Slechta et al., 2004) to produce more profound effects in the offspring of pregnant rodents than either exposure alone. These relationships varied by offspring sex. Adult male offspring demonstrated anxiety, significant memory deficits, and an increased neuroinflammatory response in the brain (e.g., decreased IL-10 levels) when co-exposed to diesel exhaust and stress during late pregnancy; female offspring of co-exposed dams showed only a slight increase in anxiety (Bolton et al., 2013). Co-exposure to stress and prenatal lead was associated with changes in dopamine and corticosterone levels in female offspring in adulthood; in males, these exposures corresponded to increased impulsive choice behaviors (Cory-Slechta et al., 2004). In the only study of stress as a modifier of pesticide exposure, Levin et al. demonstrated that when maternal rat dams were stimulated with dexamethasone during gestation to elicit a stress response and offspring were exposed to chlorpyrifos, an OP pesticide, shortly after birth, the coexposed offspring presented with greater hyperactivity, longer latency to begin feeding, and shorter feeding duration at 4-10 weeks after birth (adolescence and adulthood) compared to controls and to offspring exposed to chlorpyrifos alone. Males exposed to both factors displayed a greater increase in object exploration time and diminished locomotor activity compared to males exposed to individual treatments, whereas this effect was not found in females (Levin et al., 2014).

In the present study, we investigated the relationship between early life exposure to OPs and non-chemical stressors on cognition in children participating in CHAMACOS, a longitudinal birth cohort in the agricultural low-income community of Salinas Valley, California. We explored whether adversity modifies the associations we previously reported of prenatal OP exposure and child IQ (Bouchard et al., 2011) and, as noted in animal models, whether this association differs by child sex.

\section{Methods}

\subsection{Study participants}

The CHAMACOS study is a community-university partnership following a longitudinal birth cohort of low-income, predominantly Mexican American children in the Salinas Valley, CA, an agricultural area. Between 1999-2000, 601 women who met the following criteria were enrolled: $\geq 18$ years old; spoke English or Spanish; $<20$ weeks gestation; planned to deliver at Natividad Medical Center (the local public hospital); and were eligible for low-income health insurance (MediCal). Additional details are reported previously (Eskenazi et al., 2003). Of 536 live births, we excluded from analysis twins $(n=10)$; children with autism, Down's syndrome, hydrocephalus, and deafness $(n=4)$; and children missing DAP measurements $(n=2)$. An additional 191 children were excluded because they did not participate in the neurodevelopmental assessment at the 7-year follow-up visit. Final analyses were conducted on 329 participants. Compared to the 329 children considered in this analysis, the 191 children who did not participate were somewhat more likely to be boys, and were more likely to have mothers who had spent less than 1 year in the United States. They were not significantly different from the final sample with respect to any other key demographic characteristics or prenatal adversities considered in this analysis.

The University of California, Berkeley Committee for the Protection of Human Subjects approved all study activities. Written, informed consent was obtained for all participating women, and verbal child assent was obtained at 7 years of age.

\subsection{Procedures}

Women were interviewed twice during pregnancy, at 13 weeks and 26 weeks gestation (median). DAP metabolite concentrations were measured in maternal urine collected at each time point. Urine was aliquoted and stored at $-80^{\circ} \mathrm{C}$ prior to shipment on dry ice to the Centers for Disease Control and Prevention (CDC; Atlanta, $\mathrm{GA}$ ), where chemical analysis was conducted using gas chromatography/tandem mass spectrometry and quantified using isotope dilution calibration (Bravo et al., 2002). Each sample was measured for the presence of six nonspecific OP DAP metabolites representing three diethyl (diethylphosphate, diethylthiophosphate, and diethyldithiophosphate) and three dimethyl (dimethylphosphate, dimethylthiophosphate, dimethyldithiophophate) phosphate metabolites. These six metabolites represent byproducts from approximately $80 \%$ of total OP use in the Salinas Valley (California EPA, 2001). DAP concentrations below the limit of detection (LOD) were imputed randomly from values $<$ LOD using a log-normal distribution whose parameters were derived from maximum likelihood estimation (Lubin et al., 2004). We averaged the concentrations from the two samples; if data from one visit were missing, the non-missing value was used alone $(n=20)$. In the main analyses, we used unadjusted DAP concentrations. Creatinineadjusted DAP concentrations were used in sensitivity analyses; results did not markedly differ and therefore are not presented.

Women were interviewed again when their children were ages 6 months and $1,2,3.5,5$, and 7 years old. Interviews gathered information on demographic characteristics and varying measures of potential sources of early adversity, including average annual income, food insecurity, family structure, maternal depression, stressful life events, and family conflict. The Home Observation for the Measurement of the Environment (H.O.M.E.) (Caldwell and Bradley, 1984) was used at 6 months and 1, 2, 3.5, and 5 years during home visits, and in an office visit at 7 years to objectively assess the home learning environment and social and emotional interactions between parent and child.

We used frameworks provided by the 2000 Institute of Medicine report, From Neurons to Neighborhoods (National Research Council Institue of Medicine, 2000), the Adverse Childhood Events (A.C.E.) study (Centers for Disease Control), and other relevant literature (Alkon et al., 2014; Essex et al., 2013) to construct a priori measures of social adversity. Although we explored principal component analysis as a way to weight adversity measures, results did not provide meaningful variable reduction. Thus, all final indices were constructed by allotting one unit for each adversity experienced within a given domain. Continuous adversity indices were constructed by summing binary responses to component questions (for each component question, a binary response was constructed as $1=$ adverse or $0=$ nonadverse). In all cases, higher values reflect greater adversity among the individual adversity variables; H.O.M.E. scores were inverted to be consistent with this practice. One adversity index was constructed for each of the following six domains of experiences as detailed in Table 1: Learning Environment, Parent-Child Interaction, Maternal Adversity, Family Dysfunction, Economic Adversity, and Stressful Life Events. Additionally, we averaged the H.O.M.E. scores at each assessment point to create a measure of long-term home environment. A Total Adversity Index was constructed by summing binary responses to all unique 
Table 1

Construction of six adversity indices, including binary component variables used in each index to sum to total index score.

\begin{tabular}{|c|c|c|c|c|c|c|c|}
\hline Adversity Index & Contributing Variables ${ }^{a}$ & $\begin{array}{l}\text { Child } \\
\text { Age }\end{array}$ & $\begin{array}{l}\text { Considered } \\
\text { Adverse }\end{array}$ & Adversity Index & Contributing Variables & $\begin{array}{l}\text { Child } \\
\text { Age }\end{array}$ & Considered Adverse \\
\hline \multirow{9}{*}{$\begin{array}{l}\text { Learning } \\
\text { Environment } \\
(n=297)\end{array}$} & Maternal education & $\mathrm{BL}^{\mathrm{b}}$ & $\leq 6$ th grade & $\begin{array}{l}\text { Maternal Adversity } \\
\text { (continued) }\end{array}$ & Affective social support & $5 y$ & $\geq 2.5$ out of 5 \\
\hline & Paternal education & $\mathrm{BL}$ & $\leq 6$ th grade & & Confidant social support & $5 y$ & $\geq 2.5$ out of 5 \\
\hline & $\begin{array}{l}\text { Learning H.O.M.E. z- } \\
\text { score }^{c}\end{array}$ & $6 \mathrm{mo}$ & $>0$ & & Instrumental social support & $5 y$ & $\geq 2.5$ out of 5 \\
\hline & $\begin{array}{l}\text { Variety H.O.M.E. z- } \\
\text { score }^{\mathrm{c}}\end{array}$ & $6 \mathrm{mo}$ & $>0$ & & $\begin{array}{l}\text { Experienced everyday } \\
\text { discrimination }\end{array}$ & $5 y$ & Yes \\
\hline & $\begin{array}{l}\text { Learning H.O.M.E. z- } \\
\text { score }^{c}\end{array}$ & $1 \mathrm{y}$ & $>0$ & & $\begin{array}{l}\text { Self-rated status relative to } \\
\text { community }\end{array}$ & $7 y$ & Lowest $5 \%$ of responses \\
\hline & $\begin{array}{l}\text { Variety H.O.M.E. z- } \\
\text { score }^{\mathrm{c}}\end{array}$ & $1 \mathrm{y}$ & $>0$ & & $\begin{array}{l}\text { Self-rated status relative to } \mathrm{U} \text {. } \\
\text { S. }\end{array}$ & $7 y$ & Lowest $5 \%$ of responses \\
\hline & $\begin{array}{l}\text { Learning H.O.M.E. z- } \\
\text { score }^{c}\end{array}$ & $2 \mathrm{y}$ & $>0$ & $\begin{array}{l}\text { Family Dysfunction } \\
(\mathrm{n}=306)\end{array}$ & Family conflict score & $5 y$ & $\begin{array}{l}\geq \text { median score ( } 2 \text { out of } \\
8 \text { ) }\end{array}$ \\
\hline & $\begin{array}{l}\text { Cognitive H.O.M.E. z- } \\
\text { score }^{c}\end{array}$ & $3.5 y$ & $>0$ & & Maternal marital status & $\mathrm{BL}$ & $\begin{array}{l}\text { Not married or living as } \\
\text { married }\end{array}$ \\
\hline & $\begin{array}{l}\text { Cognitive H.O.M.E. z- } \\
\text { score }^{c}\end{array}$ & $7 y$ & $>0$ & & Maternal marital status & $2 y$ & $\begin{array}{l}\text { Not married or living as } \\
\text { married }\end{array}$ \\
\hline \multirow{16}{*}{$\begin{array}{l}\text { Parent-Child } \\
\text { Interaction } \\
(n=274)\end{array}$} & Mother return to work & $6 \mathrm{mo}$ & Yes & & Maternal marital status & $3.5 y$ & $\begin{array}{l}\text { Not married or living as } \\
\text { married }\end{array}$ \\
\hline & Physical abuse & $6 \mathrm{mo}$ & $\begin{array}{l}\text { Ever physically } \\
\text { punish }\end{array}$ & & Maternal marital status & $5 y$ & $\begin{array}{l}\text { Not married or living as } \\
\text { married }\end{array}$ \\
\hline & Physical abuse & $1 \mathrm{y}$ & $\begin{array}{l}\text { Ever physically } \\
\text { punish }\end{array}$ & & Maternal marital status & $7 y$ & $\begin{array}{l}\text { Not married or living as } \\
\text { married }\end{array}$ \\
\hline & Physical abuse & $3.5 y$ & Ever spank & & Living with non-relatives & $6 \mathrm{mo}$ & Yes \\
\hline & Physical abuse & $5 y$ & Ever spank & & Living with non-relatives & $1 \mathrm{y}$ & Yes \\
\hline & Time spent with father & $7 y$ & $\begin{array}{l}\text { Less than } 1 \mathrm{x} / \\
\text { week }\end{array}$ & & Living with non-relatives & $2 y$ & Yes \\
\hline & $\begin{array}{l}\text { Responsivity H.O.M.E. z- } \\
\text { score }^{c}\end{array}$ & $6 \mathrm{mo}$ & $>0$ & & Living with non-relatives & $3.5 y$ & Yes \\
\hline & $\begin{array}{l}\text { Avoidance H.O.M.E. z- } \\
\text { score }^{c}\end{array}$ & $6 \mathrm{mo}$ & $>0$ & & Living with non-relatives & $5 y$ & Yes \\
\hline & $\begin{array}{l}\text { Interaction H.O.M.E. z- } \\
\text { score }^{c}\end{array}$ & $6 \mathrm{mo}$ & $>0$ & & Living with non-relatives & $7 y$ & Yes \\
\hline & $\begin{array}{l}\text { Responsivity H.O.M.E. z- } \\
\text { score }^{c}\end{array}$ & $1 \mathrm{y}$ & $>0$ & $\begin{array}{l}\text { Economic Adversity } \\
(n=258)\end{array}$ & Maternal education & $\mathrm{BL}$ & $\leq 6$ th grade \\
\hline & $\begin{array}{l}\text { Avoidance H.O.M.E. z- } \\
\text { score }^{\mathrm{c}}\end{array}$ & $1 \mathrm{y}$ & $>0$ & & Paternal education & $\mathrm{BL}$ & $\leq 6$ th grade \\
\hline & $\begin{array}{l}\text { Interaction H.O.M.E. z- } \\
\text { score }^{c}\end{array}$ & $1 \mathrm{y}$ & $>0$ & & Mother returned to work & $6 \mathrm{mo}$ & Yes \\
\hline & $\begin{array}{l}\text { Responsivity H.O.M.E. z- } \\
\text { score }^{c}\end{array}$ & $2 \mathrm{y}$ & $>0$ & & Food insecurity & $\mathrm{BL}$ & $\begin{array}{l}\text { Receiving any food } \\
\text { assistance }\end{array}$ \\
\hline & $\begin{array}{l}\text { Avoidance H.O.M.E. z- } \\
\text { score }^{c}\end{array}$ & $2 y$ & $>0$ & & Food insecurity & $2 y$ & $\begin{array}{l}\text { Receiving any food } \\
\text { assistance }\end{array}$ \\
\hline & $\begin{array}{l}\text { Emotional H.O.M.E. z- } \\
\text { score }^{c}\end{array}$ & $3.5 y$ & $>0$ & & Food insecurity & $3.5 y$ & $\begin{array}{l}\text { Receiving any food } \\
\text { assistance }\end{array}$ \\
\hline & $\begin{array}{l}\text { Emotional H.O.M.E. z- } \\
\text { score }^{3}\end{array}$ & $7 y$ & $>0$ & & Food insecurity & $5 y$ & $\begin{array}{l}\text { Receiving any food } \\
\text { assistance }\end{array}$ \\
\hline \multirow{12}{*}{$\begin{array}{l}\text { Maternal } \\
\text { Adversity }^{\mathrm{d}} \\
(\mathrm{n}=284)\end{array}$} & $\begin{array}{l}\text { Feels able to influence } \\
\text { decisions }\end{array}$ & $3.5 y$ & Disagree & & Food insecurity & $7 y$ & $\begin{array}{l}\text { Receiving any food } \\
\text { assistance }\end{array}$ \\
\hline & $\begin{array}{l}\text { Satisfied with influence } \\
\text { over life }\end{array}$ & $3.5 y$ & Disagree & & Poverty status & $\mathrm{BL}$ & $\begin{array}{l}\text { At or below Federal } \\
\text { Poverty Line }^{\mathrm{d}}\end{array}$ \\
\hline & At-risk for depression & $1 \mathrm{y}$ & CES-D $\geq 16$ & & Poverty status & $6 \mathrm{mo}$ & $\begin{array}{l}\text { At or below Federal } \\
\text { Poverty Line }\end{array}$ \\
\hline & At-risk for depression & $3.5 y$ & CES-D $\geq 16$ & & Poverty status & $1 \mathrm{y}$ & $\begin{array}{l}\text { At or below Federal } \\
\text { Poverty Line }\end{array}$ \\
\hline & At-risk for depression & $7 y$ & CES-D $\geq 16$ & & Poverty status & $2 y$ & $\begin{array}{l}\text { At or below Federal } \\
\text { Poverty Line }\end{array}$ \\
\hline & Chronic depression & - & $\begin{array}{l}\text { At-risk at all } \\
\text { screens }\end{array}$ & & Poverty status & $3.5 y$ & $\begin{array}{l}\text { At or below Federal } \\
\text { Poverty Line }\end{array}$ \\
\hline & Affective social support & $\mathrm{BL}$ & $\geq 2.5$ out of 5 & & Poverty status & $5 y$ & $\begin{array}{l}\text { At or below Federal } \\
\text { Poverty Line }\end{array}$ \\
\hline & Confidant social support & $\mathrm{BL}$ & $\geq 2.5$ out of 5 & & Poverty status & $7 y$ & $\begin{array}{l}\text { At or below Federal } \\
\text { Poverty Line }\end{array}$ \\
\hline & $\begin{array}{l}\text { Instrumental social } \\
\text { support }\end{array}$ & $\mathrm{BL}$ & $\geq 2.5$ out of 5 & & Chronic poverty status & - & In poverty $\geq$ half of visits \\
\hline & Affective social support & $1 \mathrm{y}$ & $\geq 2.5$ out of 5 & $\begin{array}{l}\text { Stressful Life Events } \\
(n=315)\end{array}$ & Stressful life events scale & $5 y$ & $\geq 3$ events in past year \\
\hline & Confidant social support & $1 \mathrm{y}$ & $\geq 2.5$ out of 5 & & Stressful life events scale & $7 y$ & $\geq 3$ events in past year \\
\hline & $\begin{array}{l}\text { Instrumental social } \\
\text { support }\end{array}$ & $1 \mathrm{y}$ & $\geq 2.5$ out of 5 & & & & \\
\hline
\end{tabular}


component questions across domains. While the range varied for each index, indices were standardized for analysis as z-scores, with mean $=0$ and standard deviation $=1$, to allow for comparisons across domains of adversity. Indices are constructed as continuous measures, but analyzed as binary ( $\geq$ median and $<$ median) in models containing interaction terms.

The Wechsler Intelligence Scale, 4th edition (WISC-IV) was used to assess cognitive ability in children at 7 years of age (Wechsler, 2003), and was administered in the dominant language (English or Spanish) based on the child's performance on the oral vocabulary subtest of the Woodcock-Johnson/Woodcock-Muñoz Tests of Cognitive Ability (Woodcock and Johnson, 1990). WISC yields a Full Scale Intelligence Quotient (FSIQ), which is comprised of four subscales: Verbal Comprehension IQ(VCIQ), Perceptual Reasoning IQ (PRIQ), Working Memory IQ (WMIQ), and Processing Speed IQ (PSIQ). PSIQ and WMIQ were not completed in full during the first three months of administered interviews. Thus, 297 children have scores for FSIQ; 329 completed VCIQ and PRIQ; 298 completed PSIQ and WMIQ.

\subsection{Statistical analysis}

For individuals missing H.O.M.E. subscores at only one time point $(n=29)$, we imputed the missing value with an average of their subscore from the time points before and after. Individuals missing a H.O.M.E. subscore at only 6 months were imputed with the score received at 12 months; individuals missing a H.O.M.E. score only at 7 years were given the score received at 5 years. Average income per person was imputed using the same method $(\mathrm{n}=49)$. Individuals missing a H.O.M.E. score at more than one time point $(n=27)$, missing average income per person at more than one time point $(n=29)$, or missing any other adversity variables, which we could not reasonably impute, were excluded from models in which the missing variable was incorporated. Maternal Peabody Picture Vocabulary score was imputed when missing $(n=8)$ using the mean value from our study population $(M=100.7)$. Table 1 specifies the total sample size included in each index.

We assessed the association between each adversity index and IQ and explored effect modification of the association between maternal prenatal DAP concentration and child IQ by various adversity indices in multivariable linear regression models. Because previous literature indicated that social-environmental interactions may vary by sex (Bolton et al., 2013; Cory-Slechta et al., 2004; Levin et al., 2014), we examined the relationship between adversity and child sex on IQ, as well as a three-way interaction between adversity index, maternal DAP concentration, and child sex on IQ (relationship of maternal DAP concentrations on child IQ by sex was discussed in Bouchard et al. (Bouchard et al., 2011)). Regression coefficients were determined by building models with a two-way interaction term between prenatal DAP concentrations and adversity index scores, stratified by child sex. We report twoway interactions in order to allow comparisons between children with high and low adversity within sex, and the p-value of the three-way interaction of total DAP concentration*adversity*sex to demonstrate significance of three-way interaction; $p_{\text {interaction }}<0.2$ was considered statistically significant.

The same confounders were used as in our previous publication (Bouchard et al., 2011) that demonstrated a relationship between maternal prenatal DAP concentrations and 7-year IQ. We adjusted for maternal intelligence (as a continuous variable based on the Peabody Picture Vocabulary Test standardized score assessed
Table 2

Information on select demographic characteristics and social adversities, CHAMACOS birth cohort, Salinas, CA, 1999-2000 ( $n=329)$.

\begin{tabular}{|c|c|}
\hline Variable & $\mathrm{N}(\%)$ \\
\hline \multicolumn{2}{|l|}{ Maternal age at pregnancy (years) } \\
\hline $18-24$ & $140(42.6)$ \\
\hline $25-29$ & $109(33.1)$ \\
\hline $30-34$ & $53(16.1)$ \\
\hline $35-45$ & $27(8.2)$ \\
\hline \multicolumn{2}{|l|}{ Country of birth } \\
\hline Mexico & 282(85.7) \\
\hline U.S. & 43(13.1) \\
\hline Other & $4(1.2)$ \\
\hline \multicolumn{2}{|l|}{ Time lived in U.S. at pregnancy (years) } \\
\hline$\leq 1$ & $70(21.3)$ \\
\hline $2-5$ & $84(25.5)$ \\
\hline $6-10$ & $86(26.1)$ \\
\hline $11+$ & $53(16.1)$ \\
\hline Entire Life & $36(10.9)$ \\
\hline \multicolumn{2}{|l|}{ Language spoken at home during pregnancy } \\
\hline Mostly or only Spanish & 298(90.1) \\
\hline English and Spanish equally & $15(4.6)$ \\
\hline Mostly or only English & $13(4.0)$ \\
\hline Other & $3(0.9)$ \\
\hline \multicolumn{2}{|l|}{ Worked in agriculture during pregnancy } \\
\hline Yes & 129(39.2) \\
\hline No & $200(60.8)$ \\
\hline \multicolumn{2}{|l|}{ Maternal intelligence (PPVT score) ${ }^{a}$} \\
\hline$\leq 74$ & $106(32.2)$ \\
\hline $75-99$ & $112(34.0)$ \\
\hline$\geq 100$ & $103(31.3)$ \\
\hline Missing (imputed to mean for analysis) & $8(2.4)$ \\
\hline \multicolumn{2}{|l|}{ Child sex } \\
\hline Male & $154(46.8)$ \\
\hline Female & $175(53.2)$ \\
\hline
\end{tabular}

a Peabody Picture Intelligence Test.

when the children were 6 months old), maternal education (measured as a categorical variable of maternal education at baseline) and language of assessment (for FSIQ and VCIQ only). Models assessing the relationship between DAP concentration and 7-year IQ scores controlled for 6-month H.O.M.E. score in order to replicate previous results (Bouchard et al., 2011), whereas all other models where components of the H.O.M.E. score were not included in the adversity indices controlled for an average of H.O.M.E. scores over time (long-term H.O.M.E. score) in order to control more completely for adversity in the home. Models that included parental educational attainment in adversity indices were not adjusted for maternal education.

All analyses were performed in STATA 13.1.

\section{Results}

\subsection{Study population demographics}

Mothers of children included in this analysis were predominantly Latina; 86\% were born in Mexico and over 90\% reported speaking mostly or only Spanish at home (see Table 2). Seventy percent reported income below the Federal Poverty Line at more than half of their child's assessment visits, and almost $15 \%$ were evaluated as being at risk of clinical depression at all three

\footnotetext{
a All variables considered as binary in simple adversity indices with cut-offs shown here.

b BL, Baseline; assessed during pregnancy.

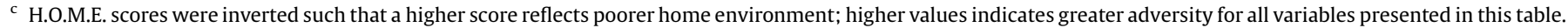

d All variables in this index refer to maternal characteristics and experiences.
} 
depression screenings. Nearly $40 \%$ worked in agriculture during pregnancy. The geometric mean for the average of the total DAP metabolite concentrations during pregnancy was 130.4 (95\% Confidence Interval (CI): 116.7, 147.0) (see Supplemental Table 1). At 7 years, the children averaged $103.7(S D=14.4$ ) for FSIQ score, $106.3(\mathrm{SD}=16.9)$ for $\mathrm{VCIQ} 102.5(\mathrm{SD}=16.1)$ for PRIQ 93.4 $(\mathrm{SD}=13.7)$ for WMIQ and $108.5(\mathrm{SD}=13.2)$ for PSIQ (see Supplemental Table 2).

\subsection{Adversity indices and IQ}

Table 3 shows the adjusted relationships between standardized adversity indices and IQ scores for all children in the cohort, and the same relationship stratified by sex. Considering boys and girls together, several types of adversity are significantly associated with decreased IQ scores. For example, adversity in the learning environment, as well as more adverse long-term H.O.M.E. scores, were significantly associated with moderate decreases in Verbal
Comprehension, Perceptual Reasoning, Working Memory, and Full-Scale IQ. Adversity in the relationship between parent and child was associated with moderate decreases in Verbal Comprehension, Working Memory, and Full-Scale IQ. Greater total adversity was associated with decreases in Verbal Comprehension and Working Memory.

Among boys, only high adversity measured by H.O.M.E. score in the early years of life was associated with lower IQ scores. A oneunit increase in the long-term H.O.M.E. adversity index corresponded to a 2.8-point decrease in VCIQ (95\% CI: -5.2, -0.4), a 2.8point decrease in PRIQ (95\% CI: $-5.6,-0.1$ ) and of borderline statistical significance, to a 2.5 -point decrease in FSIQ (95\% CI: $-4.9,0.0)$. No other categories of adverse events were significantly associated with lower IQ scores in boys.

Among girls, several domains of adversity were significantly associated with moderate decreases in IQ. A one-unit increase in long-term H.O.M.E. adversity corresponded to a 3.6-point decrease in VCIQ (95\% CI: $-5.8,-1.3$ ), a 2.7-point decrease in WMIQ (95\% CI:

Table 3

Adjusted $^{\mathrm{a}}$ association of adversity scores and WISC-IV IQ stratified by child sex, CHAMACOS birth cohort, Salinas, CA

\begin{tabular}{|c|c|c|c|c|c|c|c|c|c|c|c|}
\hline \multirow[t]{2}{*}{ IQ Test } & \multirow[t]{2}{*}{ Adversity Score } & \multirow[t]{2}{*}{$\mathrm{N}$} & \multicolumn{3}{|l|}{ Total Cohort } & \multicolumn{3}{|l|}{ Males } & \multicolumn{3}{|l|}{ Females } \\
\hline & & & $\beta(95 \% \mathrm{CI})$ & $\mathrm{p}$ & $\mathrm{N}$ & $\beta(95 \% \mathrm{CI})$ & $\mathrm{p}$ & $\mathrm{N}$ & $\beta(95 \% \mathrm{CI})$ & $\mathrm{p}$ & $\mathrm{p}_{\text {int }}^{\mathrm{b}}$ \\
\hline \multirow[t]{8}{*}{ Verbal Comprehension } & Learning Environment & 267 & $-2.0(-3.8,-0.3)$ & 0.03 & 124 & $-2.1(-4.6,0.4)$ & 0.10 & 143 & $-2.1(-4.6,0.4)$ & 0.10 & 0.57 \\
\hline & Parent-Child Interaction & 267 & $-2.1(-3.8,-0.4)$ & 0.01 & 124 & $-1.0(-3.6,1.7)$ & 0.47 & 143 & $-3.0(-5.3,-0.8)$ & $<0.01$ & 0.22 \\
\hline & Maternal Adversity & 279 & $-1.5(-3.1,0.1)$ & 0.07 & 128 & $-2.3(-4.6,0.0)$ & 0.05 & 151 & $-1.0(-3.3,1.3)$ & 0.40 & 0.39 \\
\hline & Family Dysfunction & 300 & $0.2(-1.3,1.7)$ & 0.83 & 139 & $0.9(-1.3,3.1)$ & 0.43 & 162 & $-0.8(-2.9,1.3)$ & 0.46 & 0.26 \\
\hline & Economic Adversity & 249 & $-0.6(-2.3,1.1)$ & 0.49 & 117 & $0.1(-2.4,2.6)$ & 0.93 & 132 & $-1.5(-4.0,1.0)$ & 0.23 & 0.63 \\
\hline & Stressful Life Events & 320 & $-0.9(-2.4,0.5)$ & 0.21 & 154 & $-1.7(-2.3,2.0)$ & 0.88 & 175 & $-2.0(-4.1,0.0)$ & 0.05 & 0.21 \\
\hline & Long-term H.O.M.E. Score ${ }^{c}$ & 329 & $-3 .(-4.8,-1.5)$ & $<0.01$ & 154 & $-2.8(-5.2,-0.4)$ & 0.02 & 175 & $-3.6(-5.8,-1.3)$ & $<0.01$ & 0.97 \\
\hline & Total Adversity & 225 & $-2.9(-4.7,-1.1)$ & $<0.01$ & 106 & $-2.5(-5.1,0.2)$ & 0.07 & 119 & $-3.7(-6.3,-1.2)$ & 0.01 & 0.67 \\
\hline \multirow[t]{8}{*}{ Perceptual Reasoning } & Learning Environment & 267 & $-2.1(-4.1,-0.1)$ & 0.04 & 124 & $-2.1(-4.6,0.4)$ & 0.35 & 143 & $-3.0(-5.7,-0.2)$ & 0.04 & 0.86 \\
\hline & Parent-Child Interaction & 267 & $-1.6(-3.6,0.3)$ & 0.10 & 124 & $-0.1(-2.8,3.0)$ & 0.96 & 143 & $-3.2(-5.8,-5.8)$ & 0.02 & 0.08 \\
\hline & Maternal Adversity & 279 & $0.4(-1.5,2.3)$ & 0.67 & 128 & $-0.4(-3.0,2.3)$ & 0.78 & 151 & $1.0(-1.8,3.8)$ & 0.50 & 0.63 \\
\hline & Family Dysfunction & 300 & $0.2(-1.6,2.0)$ & 0.82 & 139 & $1.6(-1.1,4.2)$ & 0.24 & 162 & $-1.3(-3.8,1.2)$ & 0.30 & 0.12 \\
\hline & Economic Adversity & 249 & $0.7(-1.3,2.7)$ & 0.50 & 117 & $1.1(-1.9,4.1)$ & 0.46 & 132 & $0.1(-2.7,2.9)$ & 0.95 & 0.77 \\
\hline & Stressful Life Events & 320 & $-1.2(-2.9,0.6)$ & 0.20 & 154 & $0.1(-2.4,2.7)$ & 0.92 & 175 & $-2.5(-4.8,-0.1)$ & 0.05 & 0.12 \\
\hline & Long-term H.O.M.E. Score & 329 & $-2.2(-3.9,-0.5)$ & $<0.01$ & 154 & $-2.8(-5.6,-0.1)$ & 0.04 & 175 & $-2.4(-5.2,0.3)$ & 0.08 & 0.72 \\
\hline & Total Adversity & 225 & $-0.8(-2.9,1.3)$ & 0.46 & 106 & $0.8(-2.2,3.8)$ & 0.60 & 119 & $-2.6(-5.7,0.4)$ & 0.08 & 0.16 \\
\hline \multirow[t]{8}{*}{ Working Memory } & Learning Environment & 240 & $-1.7(-3.4,0.1)$ & 0.07 & 114 & $-0.8(-3.2,1.7)$ & 0.53 & 126 & $-2.4(-5.1,0.2)$ & 0.07 & 0.78 \\
\hline & Parent-Child Interaction & 240 & $-2.3(-4.0,-0.6)$ & $<0.01$ & 112 & $-1.6(-4.2,1.0)$ & 0.22 & 128 & $-2.8(-5.1,-0.4)$ & 0.02 & 0.59 \\
\hline & Maternal Adversity & 250 & $-0.2(-1.8,1.5)$ & 0.84 & 115 & $-0.7(-3.0,1.6)$ & 0.56 & 135 & $0.2(-2.3,2.7)$ & 0.88 & 0.50 \\
\hline & Family Dysfunction & 270 & $-0.8(-2.4,0.7)$ & 0.30 & 126 & $-0.3(-2.6,2.0)$ & 0.77 & 145 & $-1.6(-3.7,0.6)$ & 0.15 & 0.47 \\
\hline & Economic Adversity & 225 & $-0.4(-2.1,1.3)$ & 0.63 & 107 & $-0.2(-2.8,2.3)$ & 0.86 & 118 & $-0.4(-2.8,2.1)$ & 0.76 & 0.97 \\
\hline & Stressful Life Events & 289 & $-0.4(-1.9,1.2)$ & 0.62 & 141 & $0.9(-1.2,3.0)$ & 0.42 & 157 & $-1.5(-3.7,0.8)$ & 0.19 & 0.10 \\
\hline & Long-term H.O.M.E. Score & 289 & $-2.3(-4.0,-0.6)$ & $<0.01$ & 141 & $-1.7(-4.0,0.7)$ & 0.16 & 157 & $-2.7(-5.2,-0.2)$ & 0.03 & 0.97 \\
\hline & Total Adversity & 203 & $-2.1(-3.8,-0.2)$ & 0.03 & 97 & $-1.1(-3.6,1.5)$ & 0.41 & 106 & $-3.3(-6.0,-0.6)$ & 0.02 & 0.43 \\
\hline \multirow[t]{8}{*}{ Processing Speed } & Learning Environment & 240 & $-0.6(-2.3,1.1)$ & 0.48 & 114 & $-0.2(-2.9,2.6)$ & 0.91 & 126 & $-0.8(-3.0,1.4)$ & 0.46 & 0.74 \\
\hline & Parent-Child Interaction & 240 & $-0.9(-2.6,0.8)$ & 0.32 & 112 & $0.4(-2.3,3.1)$ & 0.77 & 128 & $-1.7(-3.9,0.5)$ & 0.12 & 0.30 \\
\hline & Maternal Adversity & 249 & $-1.3(-2.9,0.4)$ & 0.12 & 115 & $-1.5(-3.9,0.9)$ & 0.21 & 134 & $-0.9(-3.2,1.4)$ & 0.45 & 0.57 \\
\hline & Family Dysfunction & 270 & $0.2(-1.4,1.8)$ & 0.81 & 126 & $1.0(-1.4,3.5)$ & 0.41 & 145 & $-0.5(-2.5,1.5)$ & 0.64 & 0.34 \\
\hline & Economic Adversity & 224 & $0.6(-1.2,2.3)$ & 0.53 & 107 & $0.6(-2.2,3.4)$ & 0.69 & 117 & $1.1(-1.2,3.4)$ & 0.34 & 0.73 \\
\hline & Stressful Life Events & 289 & $0.1(-1.4,1.7)$ & 0.88 & 141 & $-0.2(-2.6,2.1)$ & 0.84 & 156 & $-2.1(-4.3,0.1)$ & 0.06 & 0.63 \\
\hline & Long-term H.O.M.E. Score & 298 & $-1.4(-3.1,0.3)$ & 0.10 & 141 & $-0.6(-3.2,2.0)$ & 0.64 & 157 & $-2.2(-4.4,0.0)$ & 0.05 & 0.62 \\
\hline & Total Adversity & 203 & $-1.1(-3.0,0.8)$ & 0.26 & 97 & $-0.7(-3.5,2.1)$ & 0.63 & 106 & $-1.3(-4.0,1.3)$ & 0.32 & 0.97 \\
\hline \multirow[t]{8}{*}{ Full-Scale IQ } & Learning Environment & 239 & $-2.1(-3.9,-0.3)$ & 0.02 & 114 & $-1.2(-3.7,1.4)$ & 0.36 & 125 & $-2.9(-5.5,-0.3)$ & 0.03 & 0.45 \\
\hline & Parent-Child Interaction & 240 & $-1.8(-3.6,-0.1)$ & 0.04 & 112 & $-0.0(-2.8,2.7)$ & 0.98 & 128 & $-3.5(-5.9,-1.1)$ & $<0.01$ & 0.05 \\
\hline & Maternal Adversity & 249 & $-0.8(-2.4,0.9)$ & 0.37 & 115 & $-1.6(-4.0,0.8)$ & 0.20 & 134 & $-0.1(-2.6,2.3)$ & 0.91 & 0.46 \\
\hline & Family Dysfunction & 269 & $0.0(-1.6,1.5)$ & 0.96 & 126 & $1.0(-1.4,3.3)$ & 0.43 & 144 & $-1.2(-3.4,1.0)$ & 0.27 & 0.14 \\
\hline & Economic Adversity & 224 & $0.0(-1.8,1.8)$ & 0.99 & 107 & $0.4(-2.3,3.0)$ & 0.80 & 117 & $-0.1(-2.7,2.5)$ & 0.95 & 0.75 \\
\hline & Stressful Life Events & 288 & $-0.8(-2.4,0.7)$ & 0.30 & 141 & $0.1(-2.1,2.3)$ & 0.93 & 156 & $-2.1(-4.3,0.1)$ & 0.06 & 0.32 \\
\hline & Long-term H.O.M.E. Score & 297 & $-3.1(-4.7,-1.4)$ & $<0.01$ & 141 & $-2.5(-4.9,0.0)$ & 0.05 & 156 & $-3.9(-6.3,-1.5)$ & $<0.01$ & 0.59 \\
\hline & Total Adversity & 203 & $-2.0(-3.9,0.0)$ & 0.05 & 97 & $-0.7(-3.5,2.0)$ & 0.60 & 106 & $-3.5(-6.4,-0.7)$ & 0.02 & 0.23 \\
\hline
\end{tabular}

\footnotetext{
a Each row represents a separate model including the continuous adversity score indicated, and no other adversity scores. All models are adjusted for mean prenatal DAP

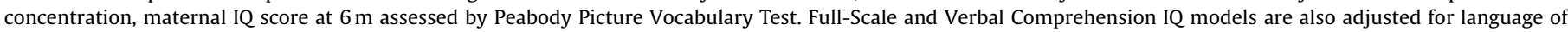

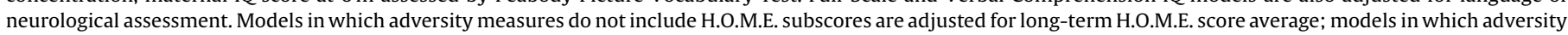
measures do not include parental educational attainment are adjusted for maternal education.

b p-int is the p-value of the two-way interaction of child sex*adversity.

c Average of standardized H.O.M.E. scores at each assessment.
} 
$-5.2,-0.2)$, and a 3.9-point decrease in FSIQ (95\% CI: $-6.3,-1.5)$. The same trend held in the relationship of increased adversity in long-term H.O.M.E. score and PRIQ and PSIQ, although these associations are of borderline significance. In addition, a one-unit increase in adverse parent-child relationship corresponded to a 3.0-point decrease in VCIQ $(95 \% \mathrm{CI}:-5.3,-1.1)$, a 3.2-point decrease in PRIQ (95\% CI: $-5.8,-0.8$ ), a 2.8-point decrease in WMIQ (95\% CI: $-5.1,-0.4$ ), and a 3.5-point decrease in FSIQ (95\% $\mathrm{CI}:-5.9,-1.1)$. A one-unit increase in adversity measured in the learning environment was associated with a 3.0-point decrease in PRIQ (95\% CI: $-5.7,-0.2)$. A one-unit increase in the total adversity score was associated with a 3.7-point decrease in VCIQ (95\% CI: $-6.3,-1.2)$, a 3.3-point decrease in WMIQ (95\% CI: $-6.0,-0.6)$, and a 3.5-point decrease in FSIQ (95\% CI: $-6.4,-0.7$ ). A one-unit increase in Stressful Life Events was associated with moderate decreases in VCIQ, PRIQ, PSIQ, and FSIQ, all with borderline statistical significance.

\subsection{Adversity, DAPs, and child sex on IQ}

In general, adverse relationships between maternal prenatal DAPs and IQ tended to be stronger in children who had experienced more adversity than those who experienced less (Table 4). In some cases these relationships varied by child sex; thus, results are presented in Table 4 for all children, and also stratified by child sex. Table 4 also presents significance of three-way interactions between adversity, DAPs, and child sex.

Considering boys and girls together, the relationship between maternal total DAPs and Verbal Comprehension is stronger among children whose mothers have experienced greater adversity $(\beta=-2.8, p=0.13)$, and among children with greater total adversity $(\beta=-3.6, p=0.09)$, as shown in Table 4 . The relationship between DAPs and Processing Speed is stronger among children with greater adversity in their learning environment $(\beta=-2.8, p=0.15)$, and with greater family dysfunction $(\beta=-2.7, p=0.18)$.

As shown in Table 4, among boys, the relationship between maternal prenatal DAP concentrations and select IQ scores was stronger among those who grew up in a poorer learning environment. For example, among boys who lived in a poorer learning environment, a 10-fold increase in DAP concentration was associated with a 13.3-point decrease in FSIQ (95\% CI -19.9, -6.7) compared to a non-significant 4.2-point increase (95\% CI -4.2 , $12.5)$ among boys who lived in better learning environment $(\mathrm{p}<0.01)$. Similar relationships were observed for VCIQ $(\beta=-9.7$ vs 5.4, $\mathrm{p}=0.06)$, PRIQ $(\beta=-9.8$ vs 7.4, $\mathrm{p}<0.01)$, and PSIQ $(\beta=-12.7$ vs $-0.2, \mathrm{p}=0.01)$. Also, among those boys with lower H.O.M.E. scores there was a stronger adverse relationship between maternal prenatal DAP concentrations and VCIQ, PSIQ, and FSIQ scores than for boys who had higher H.O.M.E. scores (VCIQ $\beta=-7.3$ vs -1.3 , $p=0.15$; PSIQ $\beta=-6.9$ vs $-4.1, p=0.17$; FSIQ $\beta=-9.6$ vs -2.4 , $\mathrm{p}=0.09$; shown in Table 4 ). No other adversity index scores modified the relationships between prenatal DAP concentrations and IQ scores in boys.

Among girls, the adverse relationship between maternal prenatal DAP concentrations and IQ was stronger among those living in poverty and those whose mothers had experienced more overall adversity (Table 4). For example, the adverse association of prenatal DAP concentration and FSIQ ( $\beta=-8.5$ vs. $-4.7, p=0.18$ ) and WMIQ ( $\beta=-5.9$ vs. $-2.6, p=0.05$ ) was stronger among girls living in households with greater than the median economic adversity compared to less than the median. Similarly, among girls whose mothers experienced greater adversity, a 10-fold increase in DAP concentration was associated with an 11.5-point decrease in VCIQ compared to only a 1.5-point decrease among girls whose mothers experienced less than the median adversity $(p=0.07)$. The relationship between prenatal DAP concentration and VCIQ was also potentiated among female children with a total adversity index score greater than the median compared to below the median $(\beta=-13.1$ vs. $-4.9, p=0.13)$.

Three-way interactions among adversity scores, total prenatal DAP concentration, and child sex were significant for IQ scores in some cases (Table 4). For example, the interaction between adversity in parent-child relationship, total DAP concentration, and child sex was significant for Perceptual Reasoning $\left(p_{\text {int }}=0.10\right)$ and Full Scale IQ $\left(p_{\text {int }}=0.06\right)$. The interaction between stressful life events, total DAP concentration, and child sex was significant for Verbal Comprehension $\left(\mathrm{p}_{\mathrm{int}}=0.19\right)$, Perceptual Reasoning $\left(\mathrm{p}_{\mathrm{int}}=\right.$ 0.12 ), and Working Memory ( $p_{\text {int }}=0.11$ ). The interaction between total adversity, total DAP concentration, and child sex was significant for Full Scale IQ ( $\left.\mathrm{p}_{\mathrm{int}}=0.13\right)$.

\section{Discussion}

This study expands upon our previous findings demonstrating an adverse association between prenatal exposure to OPs (as measured by maternal urinary DAPs) and IQ in school-age children in the CHAMACOS cohort (Bouchard et al., 2011). In the present study, we found a stronger association between prenatal OP exposure and IQ scores among children who have experienced certain adversities, and that the type of adversity that modifies this relationship may vary by sex. Among boys, poorer learning environment most strongly modified the adverse associations of prenatal OP exposure and IQ, whereas among girls, maternal and economic adversities most strongly modified this association.

Our results support previous findings demonstrating interactions between chemical and non-chemical stressors in animal studies (Bolton et al., 2013; Cory-Slechta et al., 2004; Horton et al., 2012; Levin et al., 2014), and present some of the first evidence in humans (Chen et al., 2008; Horton et al., 2012). In addition, as in previous animal studies (Bolton et al., 2013; Cory-Slechta et al., 2004), we report that the interaction between environmental and social stressors varied by offspring sex; for example, poorer learning environment most strongly modified the adverse associations of prenatal OP exposure and IQ among boys, whereas maternal and economic adversities most strongly modified this association in girls.

Associations between chemical and non-chemical stressors are biological plausible. Organophosphates target the nervous system of humans, and are associated with changes in neurotransmitters including serotonin, norepinephrine, acetylcholine, and dopamine (Slotkin and Seidler, 2007; Verma et al., 2009). The adverse effects of high allostatic load, a biological response triggered by exposure to adversity and stress, can include disrupted regulation of the normal physiologic stress response and unusually elevated stress hormone levels (Shonkoff and Garner, 2012). Our results indicate that both these exposures may target neural pathways in developing children. Future research is needed to address potential mechanisms of this interaction.

Apart from the interactions we have noted between chemical and non-chemical stressors, we have also identified that various adversities are associated with decreased cognitive function in children and may be important targets for educational and psychosocial intervention. For example, we have demonstrated that among both boys and girls, the home environment over the child's lifetime is predictive of school-age IQ. In addition, girls IQ may be predicted in part by adverse parent-child interactions, poorer learning environments, and greater overall adversity.

This analysis has several limitations. First, the population was relatively homogeneous with little variability in certain adversities. For example, most of the population lived below $200 \%$ of the Federal Poverty Level. Another limitation is that we did not collect information on certain adversities, such as sexual or emotional 


\begin{tabular}{|c|c|c|c|c|c|c|c|c|c|c|c|c|c|c|c|}
\hline \multirow[t]{2}{*}{ IQ Test } & \multirow[t]{2}{*}{ Adversity Score } & \multicolumn{3}{|c|}{ Total Cohort } & \multicolumn{5}{|c|}{ Males } & \multicolumn{5}{|c|}{ Females } & \multirow[b]{2}{*}{$\mathrm{p}_{\text {int }}{ }^{\complement}$} \\
\hline & & $\mathrm{N}$ & $\beta(95 \% \mathrm{CI})$ & $\mathrm{p}$ & $\mathrm{N}$ & $\begin{array}{l}\beta(95 \% \mathrm{CI}) \\
\text { High Adversity }\end{array}$ & $\mathrm{N}$ & $\begin{array}{l}\beta(95 \% \mathrm{CI}) \\
\text { Low Adversity }\end{array}$ & $\mathrm{p}$ & $\mathrm{N}$ & $\begin{array}{l}\beta(95 \% \mathrm{CI}) \\
\text { High Adversity }\end{array}$ & $\mathrm{N}$ & $\begin{array}{l}\beta(95 \% \mathrm{CI}) \\
\text { Low Adversity }\end{array}$ & $\mathrm{p}$ & \\
\hline \multirow[t]{8}{*}{ Verbal Compre-hension } & Learning Environment & 267 & $-1.9(-5.8,2.0)$ & 0.34 & 66 & $-9.7(-16.6,-2.7)$ & 58 & $5.4(-2.4,13.2)$ & 0.06 & 69 & $-9.2(-17.0,-1.3)$ & 74 & $-6.1(-13.6,1.4)$ & 0.91 & 0.46 \\
\hline & Parent-Child Interaction & 267 & $0.0(-4.3,4.3)$ & 0.99 & 70 & $-1.3(-10.5,7.9)$ & 54 & $-1.3(-10.7,8.1)$ & 0.83 & 74 & $-4.5(-13.2,4.1)$ & 69 & $-9.7(-16.1,-3.2)$ & 0.86 & 0.23 \\
\hline & Maternal Adversity & 279 & $-2.8(-6.4,0.8)$ & 0.13 & 66 & $-2.8(-10.4,4.8)$ & 62 & $2.3(-8.9,13.4)$ & 0.65 & 82 & $-11.5(-18.5,-4.4)$ & 69 & $-1.5(-7.9,4.9)$ & 0.07 & 0.38 \\
\hline & Family Dysfunction & 301 & $1.1(-2.7,4.9)$ & 0.56 & 66 & $-4.0(-11.7,3.7)$ & 73 & $-1.3(-9.3,6.6)$ & 0.74 & 88 & $-4.2(-10.3,1.9)$ & 74 & $-9.0(-17.2,-0.9)$ & 0.40 & 0.35 \\
\hline & Economic Adversity & 249 & $-1.9(-5.7,1.8)$ & 0.30 & 55 & $-7.7(-16.5,1.1)$ & 62 & $-0.1(-7.4,7.1)$ & 0.97 & 58 & $-9.5(-17.9,-1.2)$ & 74 & $-4.5(-11.8,2.8)$ & 0.22 & 0.41 \\
\hline & Stressful Life Events & 329 & $-2.0(-5.2,1.2)$ & 0.21 & 115 & $-4.6(-9.8,0.5)$ & 39 & $-2.4(-15.7,11.0)$ & 0.24 & 134 & $-6.4(-11.6,-1.2)$ & 41 & $-2.3(-13.0,8.5)$ & 0.36 & 0.19 \\
\hline & Long-term H.O.M.E. Score $^{d}$ & 32 & $-0.9(-4.1,2.3)$ & 0.57 & 80 & $-7.3(-14.2,-0.5)$ & 74 & $-1.3(-8.0,5.4)$ & 0.15 & 84 & $-5.4(-12.5,1.8)$ & 91 & $-6.5(-12.3,-0.7)$ & 0.61 & 0.81 \\
\hline & Total Adversity & 225 & $-3.6(-7.9,0.6)$ & 0.09 & 55 & $-3.2(-11.3,4.8)$ & 51 & $1.5(-9.6,12.7)$ & 0.29 & 54 & $-13.1(-22.1,-4.1)$ & 65 & $-4.9(-12.1,2.2)$ & 0.13 & 0.53 \\
\hline \multirow[t]{8}{*}{ Perceptual Reasoning } & Learning Environment & 267 & $-2.5(-6.9,2.0)$ & 0.30 & 66 & $-9.8(-17.4,-2.2)$ & 58 & $7.4(-2.9,17.6)$ & $<0.01$ & 69 & $-4.5(-11.9,3.0)$ & 74 & $-9.7(-19.6,0.2)$ & 0.62 & 0.86 \\
\hline & Parent-Child Interaction & 267 & $-2.5(-7.4,2.5)$ & 0.33 & 70 & $-5.1(-14.4,4.3)$ & 54 & $5.6(-6.0,17.3)$ & 0.33 & 74 & $-5.8(-14.0,2.5)$ & 69 & $-3.9(-13.5,5.8)$ & 0.62 & 0.10 \\
\hline & Maternal Adversity & 279 & $-0.4(-4.8,3.9)$ & 0.85 & 66 & $-1.8(-11.5,7.9)$ & 62 & $-1.0(-12.5,10.5)$ & 0.79 & 82 & $-5.6(-15.0,3.8)$ & 69 & $-4.3(-11.7,3.1)$ & 0.89 & 0.61 \\
\hline & Family Dysfunction & 301 & $2.4(-2.1,6.9)$ & 0.29 & 66 & $-6.0(-14.4,2.4)$ & 73 & $3.0(-6.6,12.5)$ & 0.65 & 88 & $-0.3(-6.9,6.2)$ & 74 & $-8.8(-19.2,1.7)$ & 0.12 & 0.26 \\
\hline & Economic Adversity & 249 & $-2.1(-6.5,2.3)$ & 0.34 & 55 & $-7.6(-18.1,3.0)$ & 62 & $1.1(-7.6,9.8)$ & 0.60 & 58 & $-10.8(-19.8,-1.7)$ & 74 & $-5.0(-13.7,3.6)$ & 0.47 & 0.64 \\
\hline & Stressful Life Events & 329 & $-0.9(-4.7,2.8)$ & 0.62 & 115 & $-1.2(-7.4,5.0)$ & 39 & $-12.5(-25.8,0.9)$ & 0.63 & 134 & $-2.7(-8.8,3.5)$ & 41 & $-7.2(-19.2,4.7)$ & 0.71 & 0.12 \\
\hline & Long-term H.O.M.E. Score & 329 & $-0.6(-4.3,3.2)$ & 0.77 & 80 & $-5.8(-13.3,1.7)$ & 74 & $-0.7(-9.1,7.8)$ & 0.21 & 84 & $-2.3(-9.7,5.1)$ & 91 & $-6.1(-14.0,1.8)$ & 0.52 & 0.50 \\
\hline & Total Adversity & 225 & $-1.4(-6.4,3.5)$ & 0.57 & 55 & $-5.2(-15.7,5.4)$ & 51 & $1.7(-9.1,12.6)$ & 0.23 & 54 & $-11.3(-19.7,-2.9)$ & 65 & $-4.8(-14.8,5.2)$ & 0.94 & 0.21 \\
\hline \multirow[t]{8}{*}{ Working Memory } & Learning Environment & 240 & $-1.3(-5.3,2.8)$ & 0.54 & 63 & $-8.6(-14.6,-2.6)$ & 51 & $-0.7(-9.9,8.6)$ & 0.38 & 61 & $-3.3(-10.3,3.8)$ & 65 & $-3.0(-12.2,6.2)$ & 0.86 & 0.84 \\
\hline & Parent-Child Interaction & 240 & $-1.7(-6.0,2.6)$ & 0.44 & 64 & $-6.6(-14.7,1.5)$ & 48 & $-1.0(-11.3,9.2)$ & 0.42 & 64 & $-2.6(-10.1,4.9)$ & 64 & $-3.7(-11.7,4.3)$ & 0.73 & 0.59 \\
\hline & Maternal Adversity & 250 & $0.9(-2.8,4.6)$ & 0.62 & 57 & $-4.6(-12.4,3.3)$ & 58 & $-3.8(-15.2,7.6)$ & 0.90 & 73 & $-2.4(-10.0,5.2)$ & 62 & $-5.1(-11.7,1.4)$ & 0.45 & 0.59 \\
\hline & Family Dysfunction & 271 & $-1.2(-5.2,2.8)$ & 0.56 & 60 & $-8.2(-16.2,-0.3)$ & 66 & $-2.3(-9.8,5.2)$ & 0.31 & 75 & $-1.2(-6.0,3.5)$ & 70 & $-5.7(-15.3,3.9)$ & 0.99 & 0.41 \\
\hline & Economic Adversity & 225 & $-2.1(-5.9,1.6)$ & 0.27 & 51 & $-4.7(-12.1,2.8)$ & 56 & $-7.4(-15.6,0.9)$ & 0.41 & 50 & $-5.9(-13.7,1.8)$ & 68 & $-2.6(-10.0,4.8)$ & 0.05 & 0.55 \\
\hline & Stressful Life Events & 298 & $0.5(-2.8,3.9)$ & 0.75 & 103 & $-5.2(-10.7,0.3)$ & 38 & $-6.8(-16.9,3.3)$ & 0.94 & 119 & $-1.7(-7.3,3.8)$ & 38 & $-6.3(-17.4,4.7)$ & 0.80 & 0.11 \\
\hline & Long-term H.O.M.E. Score & 298 & $-0.1(-3.4,3.2)$ & 0.96 & 72 & $-9.8(-16.0,-3.6)$ & 69 & $-0.6(-7.3,6.2)$ & 0.27 & 75 & $0.3(-6.1,6.7)$ & 82 & $-6.1(-13.1,1.0)$ & 0.41 & 0.79 \\
\hline & Total Adversity & 203 & $-2.1(-6.5,2.3)$ & 0.35 & 50 & $-4.4(-11.9,3.2)$ & 47 & $-7.0(-17.9,3.9)$ & 0.78 & 46 & $-10.3(-17.2,-3.4)$ & 60 & $0.9(-7.6,9.4)$ & 0.27 & 0.25 \\
\hline \multirow[t]{8}{*}{ Processing Speed } & Learning Environment & 240 & $-2.8(-6.7,1.0)$ & 0.15 & 63 & $-12.7(-19.5,-6.0)$ & 51 & $-0.2(-10.5,10.1)$ & 0.01 & 61 & $-4.0(-9.9,2.0)$ & 65 & $-0.9(-8.6,6.8)$ & 0.86 & 0.98 \\
\hline & Parent-Child Interaction & 240 & $1.1(-3.1,5.3)$ & 0.61 & 64 & $-6.0(-15.6,3.6)$ & 48 & $-8.7(-17.7,0.4)$ & 0.94 & 64 & $0.7(-7.4,8.8)$ & 64 & $-0.9(-6.9,5.1)$ & 0.62 & 0.32 \\
\hline & Maternal Adversity & 249 & $0.8(-2.9,4.5)$ & 0.67 & 57 & $-6.2(-16.1,3.7)$ & 58 & $-8.1(-18.0,1.8)$ & 0.68 & 73 & $0.9(-6.4,8.3)$ & 61 & $-3.1(-8.9,2.7)$ & 0.46 & 0.71 \\
\hline & Family Dysfunction & 271 & $2.7(-1.3,6.7)$ & 0.18 & 60 & $-5.6(-14.0,2.9)$ & 66 & $-4.6(-13.1,4.0)$ & 0.86 & 74 & $3.2(-2.5,8.9)$ & 71 & $-5.7(-13.1,1.7)$ & 0.13 & 0.58 \\
\hline & Economic Adversity & 224 & $-1.0(-4.8,2.8)$ & 0.61 & 51 & $-11.1(-20.6,-1.5)$ & 56 & $-7.9(-16.1,0.3)$ & 0.89 & 50 & $-3.0(-9.4,3.4)$ & 67 & $-0.7(-8.1,6.8)$ & 0.53 & 0.99 \\
\hline & Stressful Life Events & 298 & $-1.0(-4.3,2.3)$ & 0.56 & 103 & $-4.4(-10.6,1.8)$ & 38 & $-12.1(-22.5,-1.7)$ & 0.80 & 119 & $-0.2(-5.0,4.7)$ & 38 & $-3.5(-13.3,6.1)$ & 0.62 & 0.67 \\
\hline & Long-term H.O.M.E. Score & 298 & $-2.0(-5.2,1.3)$ & 0.23 & 72 & $-6.9(-14.8,1.0)$ & 69 & $-4.1(-10.9,2.7)$ & 0.17 & 75 & $0.2(-6.4,6.9)$ & 82 & $-2.6(-8.1,2.8)$ & 0.79 & 0.64 \\
\hline & Total Adversity & 203 & $1.1(-3.4,5.6)$ & 0.62 & 50 & $-9.2(-19.5,1.1)$ & 47 & $-6.4(-15.7,2.9)$ & 0.89 & 46 & $-4.7(-13.5,4.2)$ & 60 & $0.9(-5.7,7.4)$ & 0.53 & 0.96 \\
\hline \multirow[t]{8}{*}{ Full-Scale IQ } & Learning Environmeı & 239 & $-2.7(-6.7,1.4)$ & 0.20 & 63 & $-13.3(-19.9,-6.7)$ & 51 & $4.2(-4.2,12.5)$ & $<0.01$ & 60 & $-7.3(-14.8,0.2)$ & 65 & $-6.8(-15.6,2.0)$ & 0.76 & 0.68 \\
\hline & Parent-Child Interaction & 240 & $-1.1(-5.6,3.3)$ & 0.62 & 64 & $-6.4(-16.1,3.2)$ & 48 & $-1.0(-10.4,8.3)$ & 0.39 & 64 & $-3.6(-11.7,4.4)$ & 64 & $-6.3(-14.0,1.3)$ & 0.90 & 0.06 \\
\hline & Maternal Adversity & 249 & $-1.1(-4.9,2.7)$ & 0.56 & 57 & $-4.3(-14.0,5.4)$ & 58 & $-3.1(-13.5,7.2)$ & 0.85 & 73 & $-6.6(-14.4,1.1)$ & 61 & $-4.1(-10.7,2.5)$ & 0.62 & 0.51 \\
\hline & Family Dysfunction & 270 & $1.8(-2.3,5.9)$ & 0.39 & 60 & $-6.8(-14.7,1.2)$ & 66 & $-1.8(-10.3,6.7)$ & 0.58 & 74 & $-0.9(-6.0,4.3)$ & 70 & $-9.5(-18.9,-0.1)$ & 0.22 & 0.23 \\
\hline & Economic Adversity & 224 & $-2.2(-6.2,1.7)$ & 0.27 & 51 & $-10.9(-20.2,-1.6)$ & 56 & $-3.7(-11.5,4.0)$ & 0.99 & 50 & $-8.5(-16.7,-0.4)$ & 67 & $-4.7(-12.8,3.4)$ & 0.18 & 0.38 \\
\hline & Stressful Life Events & 297 & $-1.3(-4.6,2.0)$ & 0.45 & 103 & $-5.3(-10.9,0.2)$ & 38 & $-9.9(-22.4,2.7)$ & 0.40 & 118 & $-3.8(-9.0,1.5)$ & 38 & $-3.5(-16.3,9.2)$ & 0.53 & 0.30 \\
\hline & Long-term H.O.M.E. Score & 297 & $-1.2(-4.4,2.1)$ & 0.48 & 72 & $-9.6(-16.7,-2.4)$ & 69 & $-2.4(-8.8,4.1)$ & 0.09 & 75 & $-2.0(-8.8,4.7)$ & 81 & $-7.2(-13.7,-0.7)$ & 0.49 & 0.80 \\
\hline & Total Adversity & 203 & $-2.5(-7.1,2.1)$ & 0.30 & 50 & $-7.6(-17.1,1.9)$ & 47 & $-2.9(-12.8,7.0)$ & 0.23 & 46 & $-13.0(-21.5,-4.4)$ & 60 & $-2.3(-10.5,5.9)$ & 0.46 & 0.13 \\
\hline
\end{tabular}

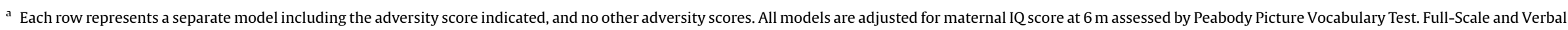

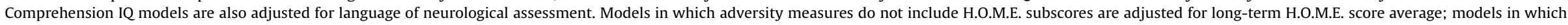
adversity measures do not include parental educational attainment are adjusted for maternal education.

${ }^{b}$ High adversity considered as greater than or equal to the median score.

c $p_{\text {int }}$ is the p-value of the three-way interaction of total DAP concentration*adversity*sex.

Pint is the p-value of the three-way interaction of total DAP c
d Average of standardized H.O.M.E. scores at each assessment. 
abuse, which are important components of the Adverse Childhood Experiences (ACE) scale (Centers for Disease Control). Lastly, although construction of adversity measures was based on a priori hypotheses, there may be more sophisticated statistical methods other than principal components analysis or simply adding adverse events, which could be used to generate measures. These measures could be based on specific effects on autonomic nervous system reactivity or on epigenetic markers and we are currently exploring these other approaches.

This study has a number of strengths. The homogeneity of the study population can reduce the influence of uncontrolled confounding. We have studied the interactive relationships between exposure to social and environmental toxicants in early life on neurodevelopment in a prospective, longitudinal study conducted in a low-income agricultural population with particularly high levels of exposure to both stress and pesticides. In comparison to other literature regarding adverse events in youth (Chen et al., 2008; Essex et al., 2013; Horton et al., 2012), this study considered a broad definition of adversity across several domains, and includes events from gestation through age 7 rather than focusing exclusively on the early postnatal years or a specific period during childhood.

In summary, this study demonstrated that the associations of OPs and child IQ at age 7 that we previously noted (Bouchard et al., 2011) are more profound in children that also experience certain social adversities, and that the specific domains of adversity that modify this association may differ in boys and girls. Those who have the highest exposure to environmental toxicants are often the same populations and individuals who experience the greatest adversity (Morello-Frosch and Shenassa, 2006). This study suggests that children with high prenatal exposure to organophosphates who also experience specific early and persistent toxic stress may be at a greater risk for adverse cognitive development.

\section{Competing financial interests}

$A B$ has participated as a volunteer member of the Board for The Organic Center, a non-profit organization that provides information for scientific research about organic food and farming. All other authors declare no competing financial interests.

\section{Funding}

This publication was made possible by U.S. Environmental Protection Agency (US EPA) (grant numbers R82670901, RD83171001, and RD83451301; Eskenazi, PI) and National Institute for Environmental Health Sciences (NIEHS) (grant numbers P01 ES009605; Eskenazi, PI). The contents of this publication are solely the authors' responsibility and do not necessarily represent the official views of the NIEHS, NIH, or EPA.

\section{Acknowledgments}

We gratefully acknowledge CHAMACOS participants and staff.

\section{Appendix A. Supplementary data}

\footnotetext{
Supplementary data associated with this article can be found, in the online version, at http://dx.doi.org/10.1016/j. neuro.2016.07.010.
}

\section{References}

Alkon, A., Boyce, W.T., Tran, L., Harley, K.G., Neuhaus, J., Eskenazi, B., 2014. Prenatal adversities and latino children's autonomic nervous system reactivity trajectories from 6 months to 5 years of age. PLoS One 9, e86283.

Bellinger, D.C., 2008. Lead neurotoxicity and socioeconomic status: conceptual and analytical issues. Neurotoxicology 29, 828-832.

Bolton, J.L., Huff, N.C., Smith, S.H., Mason, S.N., Foster, W.M., Auten, R.L., et al., 2013. Maternal stress and effects of prenatal air pollution on offspring mental health outcomes in mice. Environ. Health Perspect. 121, 1075-1082.

Bouchard, M.F., Chevrier, J., Harley, K.G., Kogut, K., Vedar, M., Calderon, N., et al., 2011. Prenatal exposure to organophosphate pesticides and iq in 7-year-old children. Environ. Health Perspect. 119, 1189-1195.

Bravo, R., Driskell, W.J., Whitehead Jr., R.D., Needham, L.L., Barr, D.B., 2002. Quantitation of dialkyl phosphate metabolites of organophosphate pesticides in human urine using gc-ms-ms with isotopic internal standards. J. Anal. Toxicol. 26, 245-252.

Caldwell, B., Bradley, R., 1984. Home observation for measurement of the environment Little Rock. University of Arkansas, AR.

California Department of Pesticide Regulation, 2012. Pesticide Information Portal, Use Data for 2012. California Department of Pesticide Regulation (accessed 09.02.15).

California EPA, 2001. Pesticide Use Reporting Summary Data. California EPA.

Centers for Disease Control, 2014. The Adverse Childhood Experiences (ace) Study. . (accessed 02.12.14) http://www.cdc.gov/violenceprevention/acestudy/.

Chen, E., Schreier, H.M., Strunk, R.C., Brauer, M., 2008. Chronic traffic-related air pollution and stress interact to predict biologic and clinical outcomes in asthma. Environ. Health Perspect. 116, 970-975.

Cory-Slechta, D.A., Virgolini, M.B., Thiruchelvam, M., Weston, D.D., Bauter, M.R., 2004. Maternal stress modulates the effects of developmental lead exposure. Environ. Health Perspect. 112, 717-730.

Engel, S.M., Berkowitz, G.S., Barr, D.B., Teitelbaum, S.L., Siskind, J., Meisel, S.J., et al., 2007. Prenatal organophosphate metabolite and organochlorine levels and performance on the brazelton neonatal behavioral assessment scale in a multiethnic pregnancy cohort. Am. J. Epidemiol. 165, 1397-1404.

Eskenazi, B.E., Bradman, A., Gladstone, E.A., Jaramillo, S., Birch, K., Holland, N., 2003. Chamacos, a longitudinal birth cohort study: lessons from the fields. J. Child. Health 1, 3-27.

Eskenazi, B., Marks, A.R., Bradman, A., Harley, K., Barr, D.B., Johnson, C., et al., 2007. Organophosphate pesticide exposure and neurodevelopment in young Mexican-American children. Environ. Health Perspect. 115, 792-798.

Essex, M.J., Boyce, W.T., Hertzman, C., Lam, L.L., Armstrong, J.M., Neumann, S.M., et al., 2013. Epigenetic vestiges of early developmental adversity: childhood stress exposure and DNA methylation in adolescence. Child Dev. 84, 58-75.

Handal, A.J., Harlow, S.D., Breilh, J., Lozoff, B., 2008. Occupational exposure to pesticides during pregnancy and neurobehavioral development of infants and toddlers. Epidemiology 19, 851-859.

Horton, M.K., Kahn, L.G., Perera, F., Barr, D.B., Rauh, V., 2012. Does the home environment and the sex of the child modify the adverse effects of prenatal exposure to chlorpyrifos on child working memory? Neurotoxicol. Teratol. 34, 534-541.

Levin, E.D., Cauley, M., Johnson, J.E., Cooper, E.M., Stapleton, H.M., Ferguson, P.L., et al., 2014. Prenatal dexamethasone augments the neurobehavioral teratology of chlorpyrifos: significance for maternal stress and preterm labor. Neurotoxicol. Teratol. 41, 35-42.

Lubin, J.H., Colt, J.S., Camann, D., Davis, S., Cerhan, J.R., Severson, R.K., et al., 2004. Epidemiologic evaluation of measurement data in the presence of detection limits. Environ. Health Perspect. 112, 1691-1696.

Morello-Frosch, R., Shenassa, E.D., 2006. The environmental riskscape and social inequality: implications for explaining maternal and child health disparities. Environ. Health Perspect. 114, 1150-1153.

National Research Council Institute of Medicine, 2000. From Neurons to Neighborhoods. National Academy Press, Washington, D.C.

Rauh, V.A., Garfinkel, R., Perera, F.P., Andrews, H.F., Hoepner, L., Barr, D.B., et al., 2006. Impact of prenatal chlorpyrifos exposure on neurodevelopment in the first 3 years of life among inner-city children. Pediatrics 118, e1845-1859.

Shonkoff, J.P., Garner, A.S., 2012. The lifelong effects of early childhood adversity and toxic stress. Pediatrics 129, e232-246.

Slotkin, T.A., Seidler, F.J., 2007. Comparative developmental neurotoxicity of organophosphates in vivo: transcriptional responses of pathways for brain cell development, cell signaling, cytotoxicity and neurotransmitter systems. Brain Res. Bull. 72, 232-274.

Sultatos, L.G., 1994. Mammalian toxicology of organophosphorus pesticides. J. Toxicol. Environ. Health 43, 271-289.

Verma, S.K., Kumar, V., Gill, K.D., 2009. An acetylcholinesterase-independent mechanism for neurobehavioral impairments after chronic low level exposure to dichlorvos in rats. Pharmacol. Biochem. Behav. 92, 173-181.

Wechsler, D., 2003. Wechsler Intelligence Scale for Children-iv: Administration and Scoring Manual. Harcourt Assessment, San Antonio, TX.

Woodcock, R.W., Johnson, M.B., 1990. Woodcock-johnson Psycho-educational Battery-revised. Riverside, Itasca, IL. 